\title{
Changes in Hox genes' structure and function during the evolution of the squamate body plan
}

\author{
Nicolas Di-Poï ${ }^{1}$, Juan I. Montoya-Burgos ${ }^{1}$, Hilary Miller ${ }^{2}$, Olivier Pourquié ${ }^{3,4} \dagger$, Michel C. Milinkovitch ${ }^{1}$ \\ $\&$ Denis Duboule $e^{1,5}$
}

\begin{abstract}
Hox genes are central to the specification of structures along the anterior-posterior body axis ${ }^{1,2}$, and modifications in their expression have paralleled the emergence of diversity in vertebrate body plans ${ }^{3,4}$. Here we describe the genomic organization of Hox clusters in different reptiles and show that squamates have accumulated unusually large numbers of transposable elements at these loci ${ }^{5}$, reflecting extensive genomic rearrangements of coding and non-coding regulatory regions. Comparative expression analyses between two species showing different axial skeletons, the corn snake and the whiptail lizard, revealed major alterations in Hox 13 and Hox 10 expression features during snake somitogenesis, in line with the expansion of both caudal and thoracic regions. Variations in both protein sequences and regulatory modalities of posterior Hox genes suggest how this genetic system has dealt with its intrinsic collinear constraint to accompany the substantial morphological radiation observed in this group.
\end{abstract}

In many animal species, Hox genes are clustered, and their expression domains, in both time and space, reflect their respective genomic order $^{1}$. Although this genetic system has been used as a paradigm in the study of the evolution of body plans ${ }^{6}$, recent studies have highlighted an unexpected diversity in Hox gene number, genomic organization and expression patterns $s^{7,8}$. In tetrapods, these genes are classified into 13 groups of paralogy and are tightly clustered at four loci: HoxA to HoxD. A clear correspondence between particular Hox groups and defined morphological boundaries along the anteroposterior axis has been documented, either by comparing expression profiles between various vertebrates or by genetic experiments in the mouse $\mathrm{s}^{1,3,4,9}$.

Vertebrate species have highly variable number of vertebrae, ranging from fewer than ten to several hundreds ${ }^{10-12}$, a parameter that is probably dependent on the speed of the segmentation clock relative to axial growth, as proposed for snakes ${ }^{13}$. Within reptiles, squamates (that is, lizards and snakes) have a large realm of morphologies, suggesting that Hox genes were modified, either in their structure or in their regulation. Previous expression analyses in snakes showed an expansion of anterior Hox gene expression along the body axis, in parallel with body plan elongation ${ }^{9}$, and revealed that collinearity was fully respected ${ }^{14}$. However, these studies involved selected genes, in the absence of genomic information. Here we describe how structural and regulatory adaptations in this gene family may have accompanied the transition towards such a body plan and suggest that the unexpected invasion of all Squamata Hox clusters by transposons might have facilitated such adaptations.

We characterized the genomic organization of posterior Hox loci in the corn snake (Pantherophis guttatus) and other reptiles, including the turtle, tuatara and several lizards, with a particular focus on repeated elements that are generally excluded from these loci in tetrapods but are abundantly present in the green anole lizard ${ }^{5}$ (Anolis carolinensis). We sequenced the posterior HoxA (from Hoxa13 to Hoxa10), HoxC (Hoxc13 to Hoxc12) and HoxD (Evx2 to Hoxd10) clusters in the corn snake (Fig. 1 and Supplementary Fig. 1), as well as the posterior HoxD cluster in other reptiles including the gecko (Gekko ulikovski) and the slow-worm (Anguis fragilis). Two nonSquamata reptile species, the turtle (Trachemys scripta) and the tuatara (Sphenodon punctatus), were used as outgroups (Fig. 1). We annotated both coding and non-coding regulatory sequences and compared them with Hox clusters from birds, mammals and amphibians (Supplementary Fig. 2).

A rather generic distribution of Hox genes was found, except in the corn snake, which lacks Hoxd12; this is similar to Xenopus (Fig. 1 and Supplementary Fig. 2a). However, we scored important modifications in conserved non-coding elements (CNEs) located at the posteriormost part of both HoxA and HoxD clusters, with the loss in all squamates (but not in non-Squamata reptiles or in other amniotes) of region $\mathrm{XII}^{15}$ located between Evx2 and Hoxd13, and the loss in corn snakes only of both region $\mathrm{XI}^{16}$, between Hoxd13 and Hoxd12, and another CNE between Hoxa13 and Hoxa11 (Fig. 1 and Supplementary Figs 1 and 2). As for the loss of Hoxd12, CNEs lost in the corn snake are also absent from Xenopus Hox clusters (Fig. 1 and Supplementary Fig. 2).

Although vertebrate Hox clusters, including those of turtle and tuatara, are relatively similar in size, all Squamata clusters were found to be substantially larger, mostly as a result of increased intronic and intergenic sizes (Fig. 1 and Supplementary Fig. 1). Such increases in length were correlated with the accumulation of transposable and other interspersed repeats, with the green anole showing the highest number. The predominant type of interspersed repeats found in anole Hox clusters consists of Penelope-like retrotransposons (PLEs) ${ }^{5}$, whereas repeats in other Squamata species are more degenerated and include short (SINE) and long (LINE) non-LTR retrotransposons, as well as DNA transposons (Fig. 1 and Supplementary Fig. 1). This atypical structure for Hox gene clusters suggests that a strong constraint was lost within this order of animals, permitting repeats to invade loci that are otherwise resistant. In turn, such repeats may have impacted significantly on both the rearrangement of coding and non-coding regulatory Hox regions and the direct regulation of Hox gene transcription in Squamata, for example through epigenetic modifications around their insertion sites ${ }^{17}$.

In amniotes, Hox expression boundaries along the rostro-caudal axis shift together with the displacement of morphological transitions between vertebral types ${ }^{3,4}$. We examined whether this correspondence was respected in snakes, despite the large expansion of 

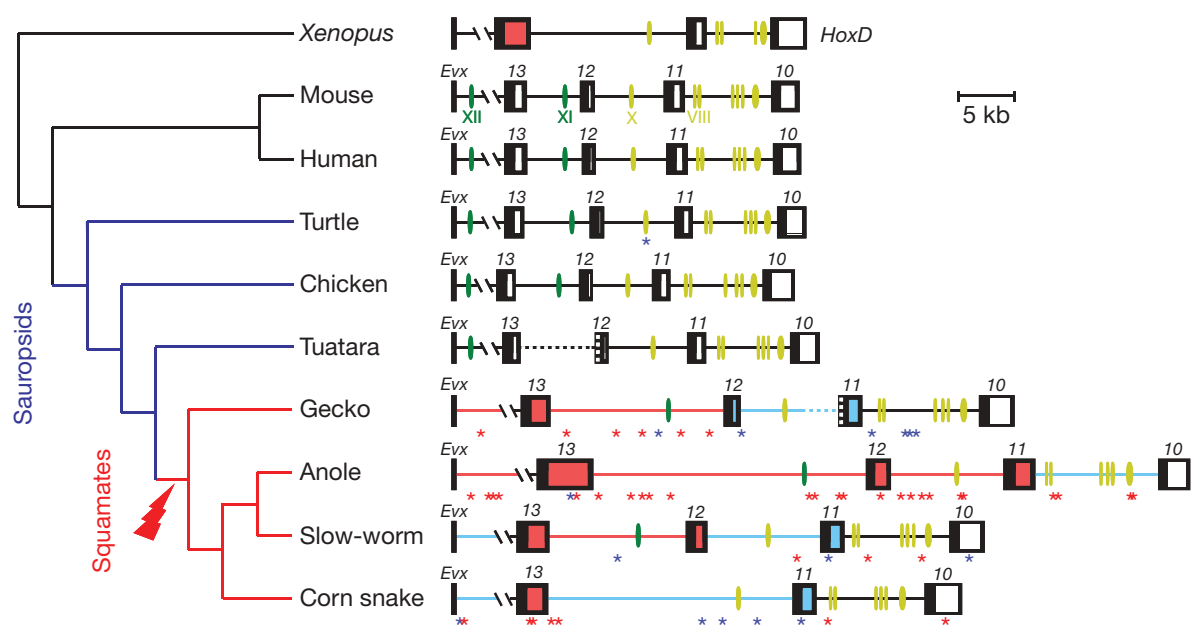

Figure 1 | Genomic organization of the posterior HoxD cluster. Schematic representation of the posterior HoxD cluster (from Evx2 to Hoxd10) in various vertebrate species. A currently accepted phylogenetic tree is shown on the left. The correct relative sizes of predicted exons (black boxes), introns (white or coloured boxes) and intergenic regions (horizontal thick lines) permit direct comparisons (right). Gene names are shown above each box. Colours indicate either a 1.5-fold to 2.0-fold (blue) or a more than 2.0-

their thoracic and caudal regions and also the transformation of the lumbo-sacral region into four cloacal vertebrae with forked ribs ${ }^{13,14}$. We investigated the expression patterns of Hox group 10 to group 13 genes by in situ hybridization in corn snake embryos at various stages. For comparison we used embryos from the whiptail lizard, which has a comparatively short body plan, with clearly identifiable regions in the pre-caudal vertebral column including 17 vertebrae bearing ribs, a single rudimentary lumbar vertebra and two sacral vertebrae. Although Hoxc13 and Hoxc12 were, as expected, expressed in the snake tail bud ${ }^{4,18}$ and subsequently in caudal mesoderm at the level of the first future caudal vertebra, neither Hoxa13 nor Hoxd13 was expressed at a detectable level in mesodermal layers during the whole process of somitogenesis in the corn snake, except for a weak and transient signal (Fig. 2 and Supplementary Figs 3 and 4). These two genes were nevertheless transcribed in the developing cloacal region at early stages, as well as in the hemipenes (Fig. 2 and Supplementary Fig. 5). In contrast, all Hox 13 genes and Hoxd12 were expressed in the lizard tail bud (Fig. 2 and Supplementary Fig. 4).

Hox11 gene expression in the snake paraxial mesoderm concerned mostly the cloacal region, in a similar manner to the sacral region in chicken ${ }^{4}$. Hoxa 11 labelled the first cloacal vertebra, whereas Hoxd11 expression matched the first caudal vertebra and Hoxc11 expression started in between. Similarly, Hoxa 11 and Hoxd11 somitic boundaries in lizard were located at the level of the first sacral and first caudal prevertebrae (Supplementary Fig. 4). Finally, we looked at the expression boundaries of all three Hox 10 paralogous genes. In other amniotes, Hox10 genes function to repress rib formation and hence they determine the thoraco-lumbar transition ${ }^{4,19}$. In snakes this morphological transition does not exist, because ribs are found until the start of the caudal region. Accordingly, Hoxd10 expression showed a sharp boundary at the level of the first caudal pre-vertebrae (Fig. 2 and Supplementary Fig. 5), similarly to Hoxd11.

In contrast, Hoxa10, which was activated much earlier in both the snake tail bud and presomitic mesoderm, showed an expression boundary well within future rib-bearing thoracic vertebrae (Fig. 2 and Supplementary Figs 3 and 5). Similarly, the expression of Hoxc10 was shifted into thoracic somitic mesoderm ${ }^{14}$ (Fig. 2 and Supplementary Fig. 5), as several Hox expression boundaries, which were shifted anteriorly at late stages of snake development and were thus quite variable throughout somitogenesis (Fig. 2 and Supplementary Fig. 4). In contrast, the expression of both lizard Hoxa10 and Hoxc10 fold (red) increase in the size of intronic (coloured boxes) or intergenic (coloured lines) regions, in comparison with the chicken reference. Major CNEs are represented by green vertical lines: light green, CNEs conserved in both mammals and sauropsids; dark green, CNEs lost in the corn snake. Gaps in the genomic sequences are indicated by dotted lines. Transposable elements are indicated with asterisks of different colours (blue for DNA transposons; red for retrotransposons).

precisely matched the unique lumbar-like pre-vertebra (Fig. 2). However, lizard Hoxd10 labelled the first caudal pre-vertebra, as in snake embryos (Fig. 2); that is, more posteriorly than in other amniotes ${ }^{4,19}$.

We compared Hox coding sequences of non-Squamata (turtles, alligator and tuatara) and Squamata (lizards and snakes) reptiles, to identify patterns of divergence and constraints across the different paralogous groups of Hox genes, which could correlate with the important morphological differences observed within these lineages ${ }^{20}$. We used likelihood models that estimated separately the rate of nucleotide substitutions either affecting (non-synonymous substitution, $d_{\mathrm{n}}$ ) or not affecting (synonymous substitution, $d_{\mathrm{s}}$ ) the amino-acid sequence for each group stem-lineage. The estimates of $d_{\mathrm{n}} / d_{\mathrm{s}}$ ratios (or $\left.\omega\right)$ are used as indicators of selective pressure acting on protein-coding sequences in the different stem-lineages, their generally low values ( $\omega$ typically less than 0.2 ; Table 1 ) thus indicating an active purifying selection throughout the evolution of vertebrate Hox genes. However, a marked increase in $\omega$ ratios was observed for several posterior Hox genes in the stem-lineage of squamates (Hoxd12 and Hoxa10), snakes (Hoxd11) or sauropsids (Hoxc10). With the exception of Hoxd11, for which three positively selected sites were identified in the stem-lineage of snakes, the higher $\omega$ ratios observed for Hoxd12, Hoxa10 and Hoxc10 are due to the relaxation of purifying selection rather than the presence of positively selected residues (Table 1).

The examination of branch lengths in the vertebrate phylogenetic tree also provides evidence for a significant relaxation of purifying selection acting on Hoxd12 in the stem lineage of squamates, as indicated by a sevenfold increase in non-synonymous substitutions (relative-rate test, $P<10^{-7}$ ), as well as within individual lizard species, leading to the loss of the gene in snakes (Supplementary Fig. 7). In addition, whereas the levels of non-synonymous substitutions are relatively low in sauropsid Hox13 genes (Supplementary Fig. 6), a relaxed selection of both Hoxa10 and Hoxc10, but not Hoxd10, is observed in the stem-lineage of squamates and sauropsids, respectively (Table 1 and Fig. 3), which is indicative of a release in functional constraints. The alignment of all Hox10 coding sequences thus revealed three strongly conserved motifs among vertebrates, and series of functionally non-equivalent amino-acid substitutions were specifically observed for Hoxa10 and Hoxc10 in snakes, whereas the Hoxd10 sequence remained well conserved (Fig. 3 and Supplementary 

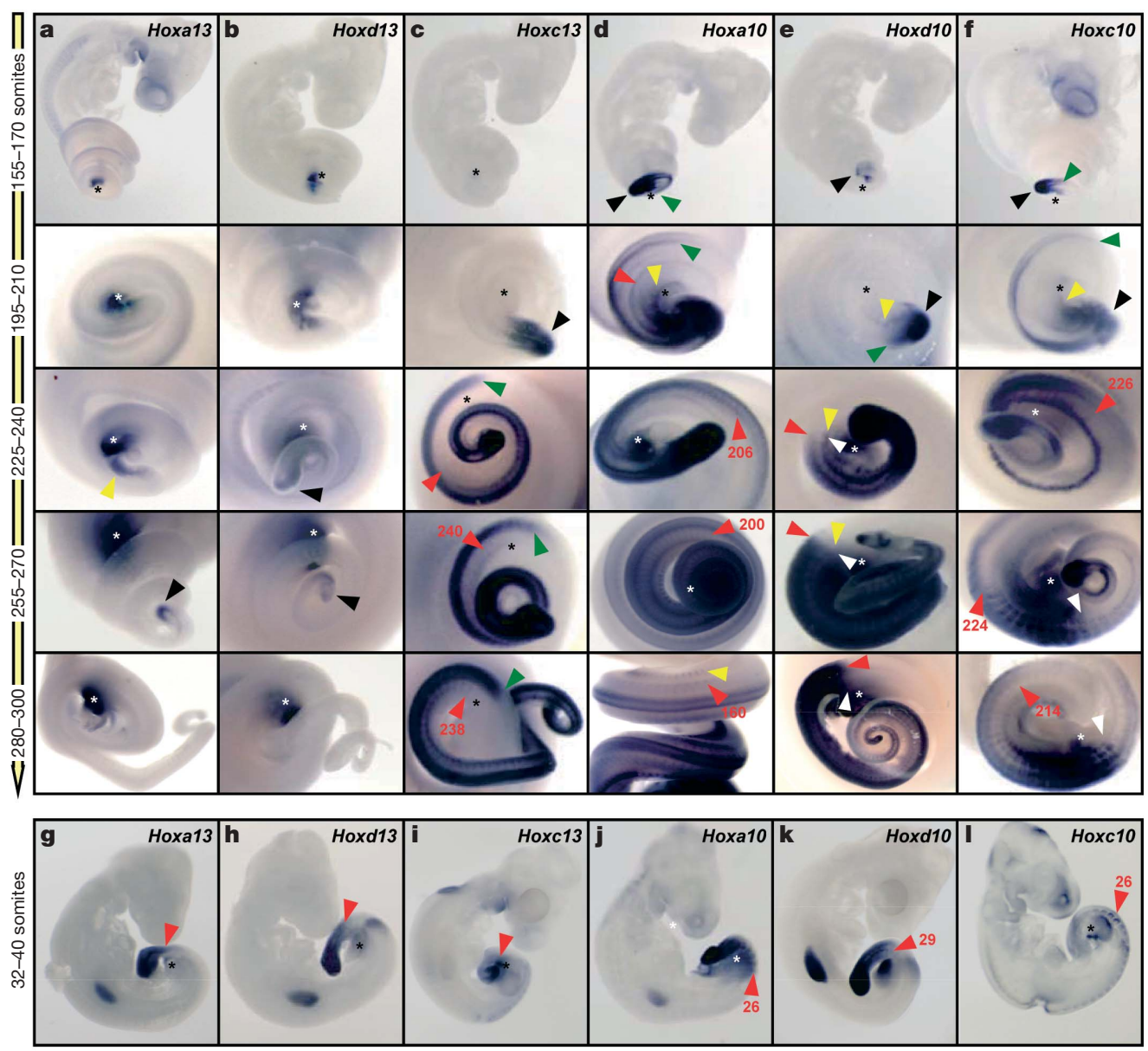

Figure 2 Expression patterns of Hox13 and Hox10 genes in snake and lizard embryos. a-f, Wholemount in situ hybridizations showing the expression of Hoxa13 (a), Hoxd13 (b), Hoxc13 (c), Hoxa10 (d), Hoxd10 (e) and Hoxc10 (f) during corn snake development (from 155 to 300 somites). Each panel shows a lateral view of the embryo (155-170 somites) or a posterior view of the somitic expression boundary at higher magnification (195-300 somites). Anterior expression boundaries are indicated with arrowheads of different colours, corresponding to distinct tissues: red, somitic mesoderm; yellow, lateral plate mesoderm; green, neural tissue; black, tail bud. The positions of the cloaca and of the fourth cloacal pre-vertebra are indicated with asterisks and white arrowheads, respectively. Numbers of somites refer to the pre-vertebral level. g-I, Expression of Hoxa13 (g), Hoxd13 (h), Hoxc13 (i), Hoxa10 (j), Hoxd10 (k) and Hoxc10 (I) in whiptail lizard embryos at 32-40 somites.
Fig. 8). We also scored strong variations in both the presence and the length of monotonic amino-acid repeats within specific coding regions of posterior Hox genes, especially between mammals and squamates (not shown). Because repeat-length mutations in Hox13 genes induce various morphological phenotypes in amniotes ${ }^{20,21}$, such natural variations, not considered by our phylogenetic analyses, may also represent a signature of adaptive changes in the coding sequence of these genes.

The importance of specific Hox paralogous groups in labelling anatomical transitions in the axial skeleton has largely been documented $^{22}$. Whereas group 10 genes specify lumbar identities by preventing the development of ribs posterior to the thorax, Hox 11 activity is required for the genesis of sacral vertebrae ${ }^{22}$. Hox 12 genes seem dispensable in this context ${ }^{16,23}$, whereas Hox 13 genes promote the termination of posterior axial structures ${ }^{24}$. Accordingly, group 11 genes in both snakes and lizards label the cloacal region, either within the region itself (Hoxc11) or at one of its ends (Hoxa11 and Hoxd11). The evolution of positively selected sites in Hox11 genes may be associated with the evolution of snake-specific functions accompanying the appearance of truncated ribs over the cloacal region (Fig. 4), in a similar manner to that proposed for Hoxa11 and the evolution of pregnancy in mammals ${ }^{25}$.

In agreement with the generic tetrapod situation, both lizard Hoxa10 and Hoxc10 label the unique vertebra without ribs, which is located immediately anterior to the sacrum (Fig. 4). However, such a situation would be problematic in snakes, in which group 11 genes are expressed immediately adjacent to ribs bearing thoracic vertebrae; that is, in structures that should normally not tolerate the ribsuppressing function of group 10 proteins. Spatial collinearity is nevertheless respected, because both Hoxa10 and Hoxc10 are expressed at more anterior levels than Hox11 genes, in areas corresponding to future rib-bearing thoracic vertebrae ${ }^{14}$ (Fig. 4). The marked relaxation in the coding sequences of these two genes suggests that the encoded proteins have lost this rib-suppressing activity in snakes to cope with the strict constraint imposed by collinearity. Hoxd10, which did not show this relaxation in protein sequence, is

Table 1 | Summary of $\omega$ ratios for posterior Hox genes in different vertebrate stem-lineages

\begin{tabular}{|c|c|c|c|c|c|c|}
\hline Gene & Amniotes & Non-eutherian mammals & Eutherian mammals & Sauropsids & Squamates & Snakes \\
\hline Hoxa13 & 0.0718 & 0.0677 & 0.0097 & 0.0231 & 0.0596 & 0.0607 \\
\hline Hoxc13 & 0.0769 & n.d. & 0.0084 & 0.0716 & 0.0500 & 0.0616 \\
\hline Hoxd13 & 0.0635 & 0.0154 & 0.0135 & 0.0293 & 0.0459 & 0.0462 \\
\hline Hoxc12 & 0.0893 & n.d. & 0.0031 & 0.0302 & 0.1762 (2 sites) & 0.0606 \\
\hline Hoxd12 & 0.3433 & 0.0931 & 0.1347 & 0.1507 (5 sites) & 0.2537 & - \\
\hline Hoxa11 & 0.0674 & 0.0912 & 0.1854 & 0.0714 & 0.0446 & 0.0848 (2 sites) \\
\hline Hoxc11 & 0.1084 & 0.0395 & 0.0808 (2 sites) & 0.1305 & 0.0182 & n.d. \\
\hline Hoxd11 & 0.1147 & 0.0431 & 0.0458 & 0.0895 & 0.0346 (1 site) & $\mathbf{0 . 2 2 3 0}$ (3 sites) \\
\hline Hoxa10 & 0.0348 & 0.0804 (2 sites) & 0.1179 & 0.1491 & 0.3831 & 0.1251 \\
\hline Hoxc10 & 0.2068 & n.d. & 0.1611 & 0.2389 & 0.0345 & 0.1031 \\
\hline Hoxd10 & 0.0896 & 0.1402 & 0.0955 & 0.0308 & 0.0887 & 0.1019 \\
\hline
\end{tabular}

Amino-acid sites under positive selection are indicated within brackets; $\omega$ ratios more than 0.2 are in bold. n.d., not determined. 

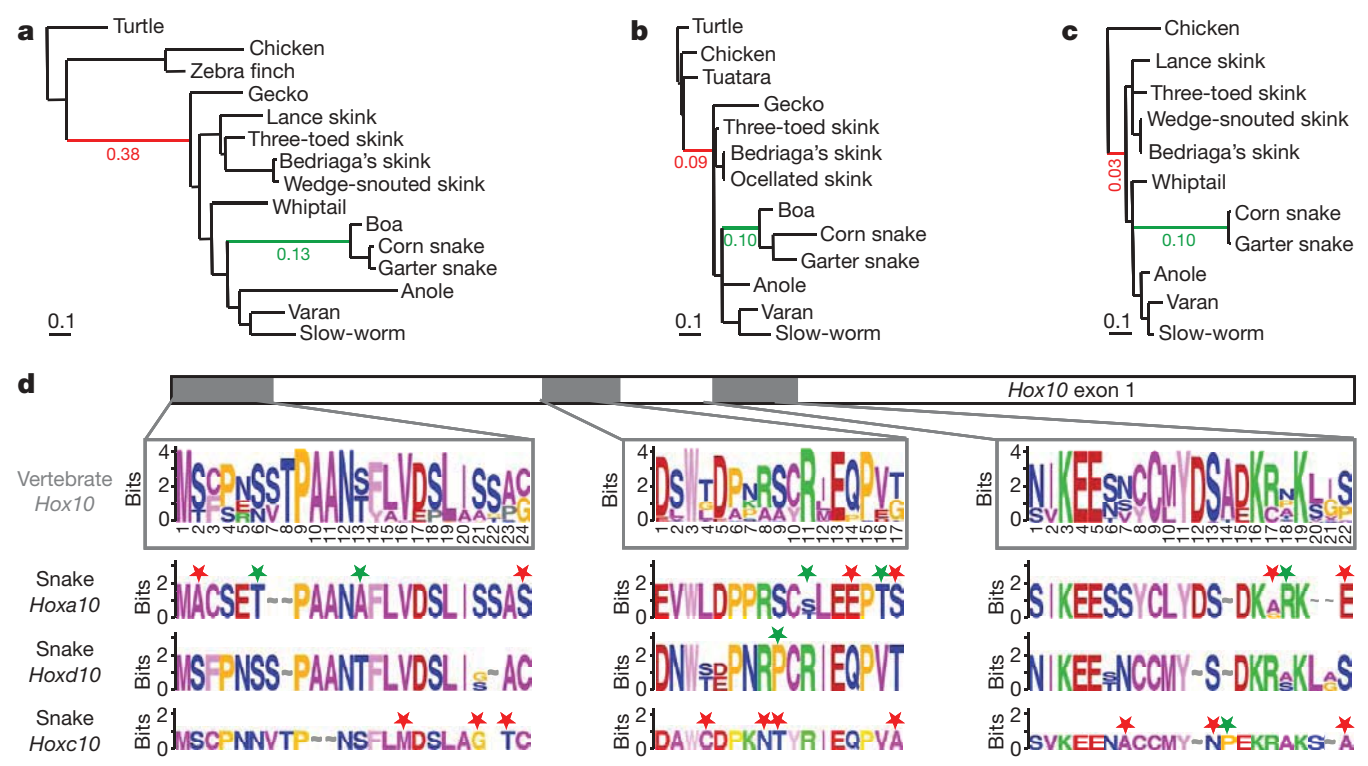

Figure 3 | Comparison of vertebrate HOX10 protein sequences. a-c, Phylogenetic trees of sauropsid Hoxa10 (a), Hoxd10 (b) and Hoxc10 (c) exon 1. Branch lengths are proportional to the number of nonsynonymous substitutions per site. Values for $\omega$ are indicated for the stemlineage of squamates (red) and snakes (green). d, Amino-acid substitutions in the first exon of Squamata Hox10 paralogous genes. Grey boxes indicate the locations of strongly conserved amino-acid motifs among all tested

expressed in snake at the same antero-posterior level as Hoxd11, that is, the only possible position allowed by the combined constraints of keeping in register with spatial collinearity and maintaining a ribsuppressing activity.

In contrast to all other amniotes analysed so far ${ }^{3,4}$, including the whiptail lizard, neither Hoxa13 nor Hoxd13 is expressed within the post-cloacal tail bud and somitic mesoderm in the snake. Because Hox13 genes are the most posterior group in tetrapods and thus

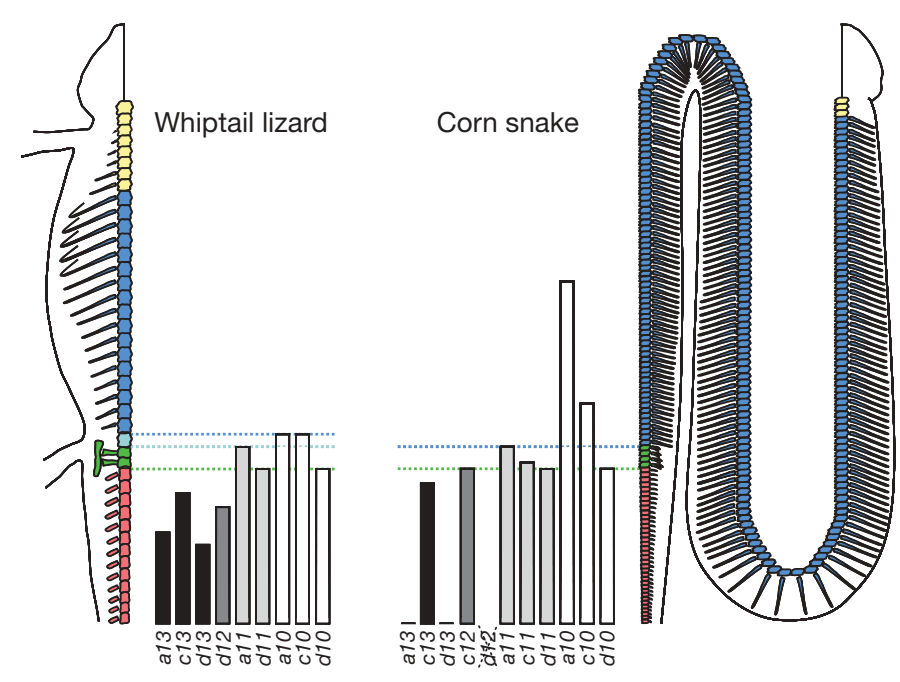

Figure 4 | Evolutionary modifications of the posterior Hox system in the whiptail lizard and corn snake. The positions of Hox expression domains along the paraxial mesoderm of whiptail lizard (32-40 somites, left) and corn snake (255-270 somites, right) are represented by black (Hox13), dark grey (Hox12), light grey (Hox11) and white (Hox10) bars, aligned with coloured schemes of the future vertebral column. Colours indicate the different vertebral regions: yellow, cervical; dark blue, thoracic; light blue, lumbar; green, sacral (in lizard) or cloacal (in snake); red, caudal. Hoxc11 and Hoxc12 were not analysed in the whiptail lizard. Note the absence of Hoxa13 and Hoxd13 from the corn snake mesoderm and the absence of Hoxd12 from the snake genome. vertebrate Hox 10 genes. The three snake sequence logos show variations within individual Hoxa10, Hoxd10 and Hoxc10 genes. Amino acids are colour-coded by physicochemical properties. The size of the letters is proportional to the number of species with the given amino acid. Stars indicate the locations of sites substituted either in all squamates (red stars) or in snakes only (green stars).

determine structures located at the extremities of body axes, it has been proposed that they may function to terminate the patterning process $^{24}$. Consequently, it is tempting to associate the absence of Hoxa13 and Hoxd13 expression with the elongation of the caudal region in snakes, perhaps by delaying the progressive shrinking of presomitic mesoderm, which seems to control the termination of somitogenesis ${ }^{13}$. Accordingly, a larger number of cell generations is estimated to be required to build the snake axis than in the chicken ${ }^{13}$. For both Hoxa13 and Hoxd13, modifying the transcriptional regulation was probably a more parsimonious solution than relaxing protein sequences, because of their additional functions in the development of cloaca and hemipenes ${ }^{26}$. In contrast, Hoxc13 was expressed in the tail bud along with Hoxc12, as expected from patterns in other tetrapods, which may be the minimum required for a difference to emerge between cloacal and post-cloacal vertebral morphologies in snakes.

Our results suggest that snakes, like mammals, use the full realm of group 11 genes to determine the cloacal region, which is critical for both vertebrate axial patterning and physiology. However, anteriorly and posteriorly to this region, a simplified Hox code seems to be at work: first, only Hoxc13 (not Hoxa13 or Hoxd13) and Hoxc12 (not Hoxd12) may help to terminate body elongation, and second, only Hoxd10 may have conserved the rib-suppressing activity. Snakes may therefore implement a smaller number of Hox gene functions in these posterior areas. Such a relaxation in functional redundancy, when compared with that in other tetrapods (Fig. 4), either at the level of transcription or at that of coding sequences, may have been a prerequisite for the marked modifications that occurred in the snake axial skeleton. Anterior shifts in the expression patterns of Hoxa10 and Hoxc10 may still correlate with the presence of distinct structures and/or various size of ribs or vertebral processes along the anteroposterior axis, despite the apparent homogeneity of the snake thoracic region ${ }^{11}$.

Consistent with previous phylogenetic analyses ${ }^{27}$ is our demonstration that the transition from short lizard-like to elongated snakelike body forms involved progressive changes in the Squamata body plan, which can be somewhat prefigured in lizards. For example, the expression of Hoxd10 in the whiptail lizard does not correspond to 
the lumbo-sacral transition, as in other amniotes ${ }^{4,19}$, yet it coincides with that found in snakes (Fig. 4), as if the function of Hoxd10 at the caudo-cloacal transition (the end of rib-bearing vertebrae) was already implemented in lizards. Similarly, although the loss of the CNE XII in the lizard HoxD loci may not have drastically modified posterior Hoxd gene expression, the additional loss of region XI in the snake may have elicited significant effects ${ }^{15}$.

Elongated body shapes have evolved independently in different vertebrate groups such as eels, salamanders, caecilians, lizards and snakes $^{11,12,14}$, and preliminary observations suggest that these various species use similar processes both for segmentation and for segmental identity ${ }^{13,28}$. Future studies integrating the period of the somitic clock with Hox gene expression in vertebrates with a specific elongation of either their thorax (for example burrowing snakes and caecilians) or their post-sacral region (for example varanoids and salamanders) will confirm whether natural selection has indeed exploited the flexibility of similar developmental mechanisms. The fact that limb reduction is commonly found along with trunk elongation suggests that posterior Hox genes may have had major functions in coordinating the emergence of these novel forms ${ }^{9,14}$, because extensive genetic analyses in mice have demonstrated the critical importance of these genes in limb development and evolution ${ }^{29}$.

\section{METHODS SUMMARY}

Hox genes were cloned after PCR amplification from reptile genomic DNA, using various combinations of degenerated primers corresponding to sequences that are highly conserved in vertebrates. Full posterior Hox clusters were amplified by long-range PCRs with the Expand Long Template PCR System (Roche) and LA Taq DNA polymerase (TaKaRa Bio), and were sequenced to high coverage (more than 20-fold) with the 454 FLX sequencing technology (Roche). Annotation of vertebrate Hox clusters was generated from sequence alignments and nucleotide BLAST searches at the University of California Santa Cruz and the National Centre for Biotechnology Information. To further validate exon boundaries, putative coding regions were predicted with GenScan. Interspersed repeats were identified and classified with both Censor and Repeat-Masker, using nucleotide sequences and default parameters.

In all phylogenetic analyses, exonic sequences were aligned using ClustalW and manually rearranged using the Bioedit alignment software. Estimation of $\omega$ was performed with the maximum-likelihood (ML) method as implemented in CODEML in PAML v.4.2 (ref. 30) and using branch-specific evolutionary models. The imposed species tree is in accord with current knowledge on vertebrate phylogeny. Selective pressures acting on sites along specific branches of the phylogenetic tree were assessed with branch-site models, and amino-acid sites under positive selection were identified with the Bayes Empirical Bayes (BEB) method (threshold probability of more than 0.90 under model A).

Whole-mount in situ hybridizations were performed with standard procedures at a hybridization temperature of $68^{\circ} \mathrm{C}$ and digoxigenin-labelled riboprobes (Supplementary Table 1). Snake and lizard embryos were carefully staged by somite counting as described ${ }^{6,13}$, using standard or three-dimensional optical projection tomography imaging, and gene expression boundaries reported here correspond to the pre-vertebral level. For histology, embryos after whole-mount in situ hybridization were fixed in $4 \%$ paraformaldehyde, cryoprotected in 30\% sucrose, embedded in OCT compound and cryosectioned at $20 \mu \mathrm{m}$.

\section{Received 9 October; accepted 18 December 2009.}

1. Kmita, M. \& Duboule, D. Organizing axes in time and space; 25 years of colinear tinkering. Science 301, 331-333 (2003)

2. Deschamps, J. \& van Nes, J. Developmental regulation of the Hox genes during axial morphogenesis in the mouse. Development 132, 2931-2942 (2005).

3. Gaunt, S. J. Conservation in the Hox code during morphological evolution. Int. J. Dev. Biol. 38, 549-552 (1994).

4. Burke, A. C., Nelson, C. E., Morgan, B. A. \& Tabin, C. Hox genes and the evolution of vertebrate axial morphology. Development 121, 333-346 (1995).

5. Di Poi, N., Montoya-Burgos, J. I. \& Duboule, D. Atypical relaxation of structural constraints in Hox gene clusters of the green anole lizard. Genome Res. 19, 602-610 (2009).

6. Duboule, D. The rise and fall of Hox gene clusters. Development 134, 2549-2560 (2007).
7. Garcia-Fernandez, J. The genesis and evolution of homeobox gene clusters. Nature Rev. Genet. 6, 881-892 (2005).

8. Lemons, D. \& McGinnis, W. Genomic evolution of Hox gene clusters. Science 313, 1918-1922 (2006).

9. Cohn, M. J. \& Tickle, C. Developmental basis of limblessness and axial patterning in snakes. Nature 399, 474-479 (1999).

10. Hoffstetter, R. \& Gasc, J. P. in Biology of the Reptilia (eds Gans, C., d'A. Bellair, A. \& Parsons, T. S.) Vol. 1, 201-310 (Academic, 1969).

11. Romer, A. S. Osteology of the Reptiles (Krieger Publishing Co., 1997).

12. Duellman, W. E. \& Trueb, L. Biology of Amphibians (Johns Hopkins University Press, 1994).

13. Gomez, C. et al. Control of segment number in vertebrate embryos. Nature 454, 335-339 (2008)

14. Woltering, J. M. et al. Axial patterning in snakes and caecilians: evidence for an alternative interpretation of the Hox code. Dev. Biol. 332, 82-89 (2009).

15. Kmita, M., Tarchini, B., Duboule, D. \& Herault, Y. Evolutionary conserved sequences are required for the insulation of the vertebrate Hoxd complex in neural cells. Development 129, 5521-5528 (2002).

16. Herault, Y., Beckers, J., Kondo, T., Fraudeau, N. \& Duboule, D. Genetic analysis of a Hoxd-12 regulatory element reveals global versus local modes of controls in the HoxD complex. Development 125, 1669-1677 (1998).

17. Feschotte, C. \& Pritham, E. J. DNA transposons and the evolution of eukaryotic genomes. Annu. Rev. Genet. 41, 331-368 (2007).

18. Godwin, A. R. \& Capecchi, M. R. Hoxc13 mutant mice lack external hair. Genes Dev. $12,11-20$ (1998).

19. Carapuço, M., Novoa, A., Bobola, N. \& Mallo, M. Hox genes specify vertebral types in the presomitic mesoderm. Genes Dev. 19, 2116-2121 (2005).

20. Kohlsdorf, T. et al. A molecular footprint of limb loss: sequence variation of the autopodial identity gene Hoxa-13. J. Mol. Evol. 67, 581-593 (2008).

21. Muragaki, Y., Mundlos, S., Upton, J. \& Olsen, B. Altered growth and branching patterns in synpolydactyly caused by mutations in Hoxd13. Science 272, 548-551 (1996).

22. Wellik, D. M. \& Capecchi, M. R. Hox10 and Hox11 genes are required to globally pattern the mammalian skeleton. Science 301, 363-367 (2003).

23. Suemori, H. \& Noguchi, S. HoxC cluster genes are dispensable for overall body plan of mouse embryonic development. Dev. Biol. 220, 333-342 (2000).

24. Young, T. et al. Cdx and Hox genes differentially regulate posterior axial growth in mammalian embryos. Dev. Cell 17, 516-526 (2009).

25. Lynch, V. J. et al. Adaptive changes in the transcription factor HoxA-11 are essential for the evolution of pregnancy in mammals. Proc. Natl Acad. Sci. USA 105, 14928-14933 (2008).

26. Scott, V., Morgan, E. A. \& Stadler, H. S. Genitourinary functions of Hoxa13 and Hoxd13. J. Biochem. 137, 671-676 (2005).

27. Wiens, J. J. \& Slingluff, J. L. How lizards turn into snakes: a phylogenetic analysis of body-form evolution in anguid lizards. Evolution 55, 2303-2318 (2001).

28. Raynaud, A. Preliminary data on the body lenghthening and somitogenesis in young embryos of Anguis fragilis L. and of Lacerta viridis Laur. Bull. Soc. Hist. Nat. Toulouse 130, 47-52 (1994).

29. Zakany, J. \& Duboule, D. The role of Hox genes during vertebrate limb development. Curr. Opin. Genet. Dev. 17, 359-366 (2007).

30. Yang, Z. PAML 4: phylogenetic analysis by maximum likelihood. Mol. Biol. Evol. 24, 1586-1591 (2007).

Supplementary Information is linked to the online version of the paper at www.nature.com/nature.

Acknowledgements We thank A. Schmitz for sharing squamate tissues; A. Debry and F. Chabaud for technical assistance; and members of the Duboule and Milinkovitch laboratories for discussions and reagents. This work was supported by funds from the University of Geneva and the Federal Institute of Technology in Lausanne, the Swiss National Research Fund, the National Research Center (NCCR) 'Frontiers in Genetics', the EU programme 'Crescendo' and the ERC grant SystemsHox.ch (to D.D.).

Author Contributions N.D.P. and D.D. designed the experiments and analysed the data. N.D.P. performed the experiments except those involving the tuatara, which were conducted by H.M. J.I.M.B. performed the phylogenetic analyses. M.C.M. produced and prepared snake embryos and O.P. provided snake and lizard embryos. N.D.P. and D.D. wrote the paper, and all co-authors contributed in the form of discussion and critical comments.

Author Information Sequences of genes described in this paper are deposited in GenBank under accession numbers GU320304 to GU320335. Reprints and permissions information is available at www.nature.com/reprints. The authors declare no competing financial interests. Correspondence and requests for materials should be addressed to D.D. (denis.duboule@unige.ch or denis.duboule@epfl.ch). 